\title{
ANÁLISIS Y SÍNTESIS DE LAS CONSONANTES NASALES
}

\author{
María José Albalá \\ Consejo Superior de Investigaciones Científicas
}

\section{INTRODUCCIÓN}

El sistema fonológico del español presenta tres fonemas nasales: uno bilabial $/ \mathrm{m} /$, otro linguoalveolar $/ \mathrm{n}$ / y otro linguopalatal $/ \mathrm{n} /$, que funcionan como tales en posición silábica explosiva. En posición implosiva, la oposición entre $/ \mathrm{m} / \mathrm{y} / \mathrm{n} /$ se neutraliza y da como resultado el archifonema $/ \mathrm{N} /$. En el plano de las realizaciones, el segmento nasal postnuclear se asimila a la consonante siguiente, lo que da lugar a la aparición de varios alófonos según el lugar de articulación de esta última consonante: bilabial [m], labiodental [m], interdental [n], dental [n], alveolar [n], palatalizado [n] y velar [n].

Jakobson, Fant y Halle ${ }^{1}$ revelaron que los rasgos distintivos de un sonido poseen ciertos correlatos acústicos y articulatorios que no se presentan a nuestras manos como unidades indivisas, sino como manojos de índices: del mismo modo que el fonema está constituido por un haz de rasgos distintivos, éstos a su vez se manifiestan por medio de sus correlatos físicos y fisiológicos.

Pues bien, nos proponemos aquí estudiar los índices articulatorios y acústicos de las consonantes nasales españolas, a pesar de que esta disección es artificiosa: las distintas propiedades se encuentran tan intimamente interrelacionadas que sólo deshaciendo el lienzo es posible examinar separadamente sus hilos. Al final trataremos de entrelazarlos de nuevo con el objeto de devolverles su trama originaria.

1 Preliminaries to Speech Analysis. The Distinctive Features and their Correlates, Massachussets, 1952. 


\section{RASGOS ARTICULATORIOS}

Las nasales como clase de sonidos presentan los siguientes indices articulatorios: en primer lugar, un carácter oclusivo, puesto que la corriente de aire que procede de las cavidades infraglóticas encuentra en la boca un momento de cierre total de los órganos; en segundo término, una naturaleza nasal —que les da su nombre—, dado que en su articulación se produce un descenso del velo del paladar, movimiento que permite la salida del aire a través de la nariz, y por último, el rasgo sonoro, puesto que en su producción hay vibración de las cuerdas vocales.

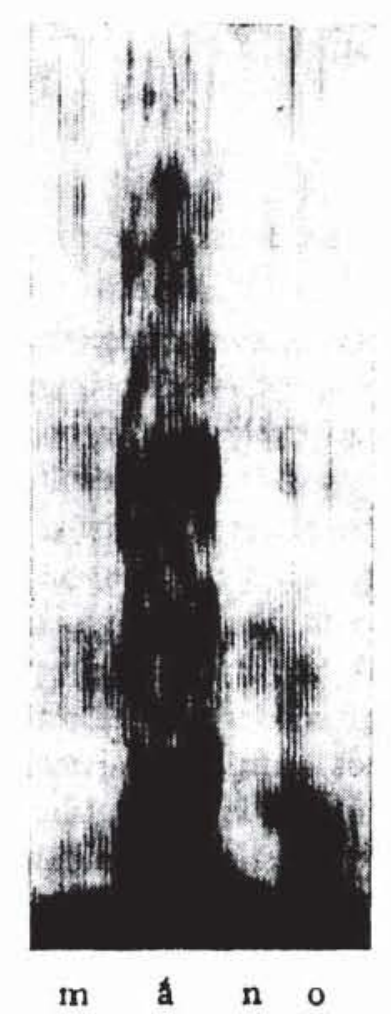

Figura $1 .-/$ máno/ (voz natural)

Además, cada consonante nasal presenta sus propias caracteristicas en función de su lugar de articulación, es decir, según la oclusión se realice por el contacto entre ambos labios, o bien entre la lengua y la zona dental, alveolar, palatal o velar ${ }^{2}$.

2 Véanse dibujos de las articulaciones nasales en A. Quilis, El comentario fonolbgico $y$ fonético de textos, Madrid, Arco libros, 1985, pág. 55. 


\section{RASGOS ACÚSTICOS}

En el sonograma de una consonante nasal (figura 1) aparecen varias bandas de resonancia, de intensidad variable, entre las cuales hay algunas concentraciones de energía que constituyen verdaderos formantes. En esta resonancia deben encontrarse, pues, los índices acústicos tanto del modo de articulación nasal como de los distintos lugares articulatorios. Con el objeto de acotar estos índices, hemos realizado un estudio experimental en dos fases sucesivas de análisis y sintesis.

\subsection{Estudio EXPERIMENTAL}

Hemos analizado, en primer lugar, 712 sonogramas procedentes de grabaciones hechas a hombres y mujeres de origen geográfico castellano. Registramos en cinta magnética la voz de ocho informantes, cada uno de los cuales leyó - con la mayor naturalidad posible y a una velocidad de elocución normal- 89 palabras incluidas en frases portadoras, en cuyo conjunto aparecían los tres fonemas nasales y sus alófonos, en las diferentes posiciones silábicas que son posibles (inicial, medial en sílaba tónica y átona e implosiva) y en todos los contextos vocálicos. Después, nos hemos servido de un programa sintetizador realizado en la Escuela Técnica Superior de Ingenieros de Telecomunicación de la Universidad Politécnica de Madrid según el modelo de Klatt ${ }^{3}$. Con su ayuda, hemos ido perfilando los datos procedentes del análisis hasta conseguir una sintesis de los tres fonemas nasales, que luego hemos sometido a una prueba de reconocimiento por parte de 20 informantes.

Los métodos de síntesis, como es conocido, parten de una codificación de la onda sonora gracias a la cual ésta puede manipularse y posteriormente descodificarse: primero tiene lugar — por medio de un proceso de muestreo y cuantificación - una conversión analógico-digital de la onda continua en elementos discretos que pueden ser modificados; y después, el proceso inverso: una conversión digital-analógica para obtener de nuevo la señal acústica 4 . El programa de Klatt utiliza la técnica paramétrica, que permite codificar la onda sonora analizada en sus distintos parámetros. Para seleccionar estos últimos se toma como punto de referencia el modelo de producción del habla

3 Cfr. «Software for a cascade / parallel formant synthesizer», Journal of the Acous. tical Society of America, 67, 1980, págs. 971-995.

4 Sobre las técnicas de sintesis, cfr. A. Quilis, «El empleo de los ordenadores en la investigación fonética», Lingüística española actual, III/2, 1981, págs. 197-219, у Joaquim Llisterri Boix, «La síntesis del habla: estado de la cuestión», Procesamiento del lenguaje natural, 6, 1988, págs. 19-41. 
de Fant ${ }^{5}$, según el cual en la articulación de un sonido lingüístico son necesarios dos elementos, una fuente y un filtro. El aire que procede de las cavidades infraglóticas, en efecto, encuentra en su salida hacia el exterior un obstáculo que es la fuente del sonido: las cuerdas vocales en vibración - cuando se trata de un sonido periódico- o - en los sonidos aperiódicosun estrechamiento o una oclusión en la vía de salida del aire, consecuencia, respectivamente, de la aproximación o del contacto de los órganos articulatorios en la cavidad bucal. Los sonidos se producen, así pues, en la glotis (fuente periódica) o en la boca (fuente aperiódica); pero las cavidades supraglóticas - la faringe y los resonadores bucal y nasal- alteran la estructura espectral que presenta el sonido en la fuente, es decir, actúan como filtro en una precisa función de transferencia. Pues bien, las propiedades de la fuente y la función de transferencia propia del filtro determinan en cada caso los parámetros que se toman para codificar la onda sonora ${ }^{6}$.

El programa de síntesis es capaz de simular los dos elementos: por un lado, las distintas fuentes de sonido y, por otro, las diferentes funciones de transferencia del tracto vocal. Éstas se imitan por medio de resonadores que filtran las frecuencias de los formantes, cuya conexión puede configurarse en serie o en paralelo.

El sintetizador de Klatt se caracteriza por utilizar ambos tipos de confi-
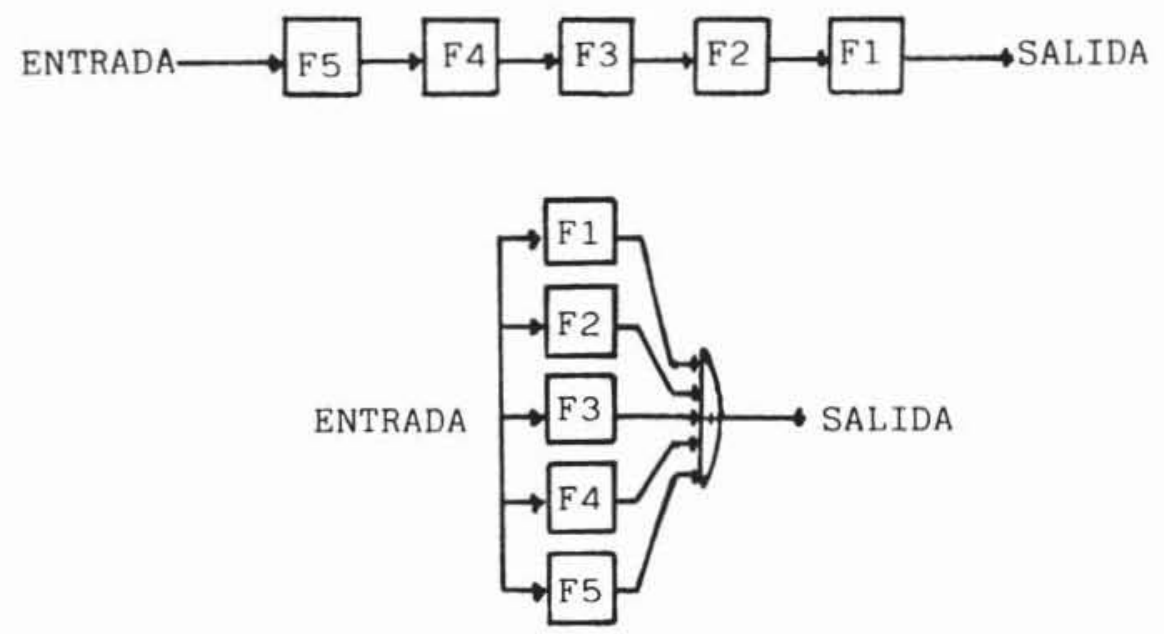

Figura 2.-Conexión en serie y conexión en paralelo ?

- Acoustic Theory of Speech Production (2." ed.), The Hague, Mouton, 1970.

- A la fuente del sonido y a la función de tranferencia hay que añadir un tercer ele. mento, la radiación característica (cfr. Klatt, art. cit., págs. 972 y 983), que permite la propagación del sonido desde los labios.

7 Cfr. Klatt, art. cit., pág. 972, figura 3. 
guraciones: la función de transferencia del tracto vocal para la fuente periódica se imita por medio de filtros conectados en serie, en tanto que la que corresponde a la fuente aperiódica se simula a través de una conexión en paralelo ${ }^{8}$.

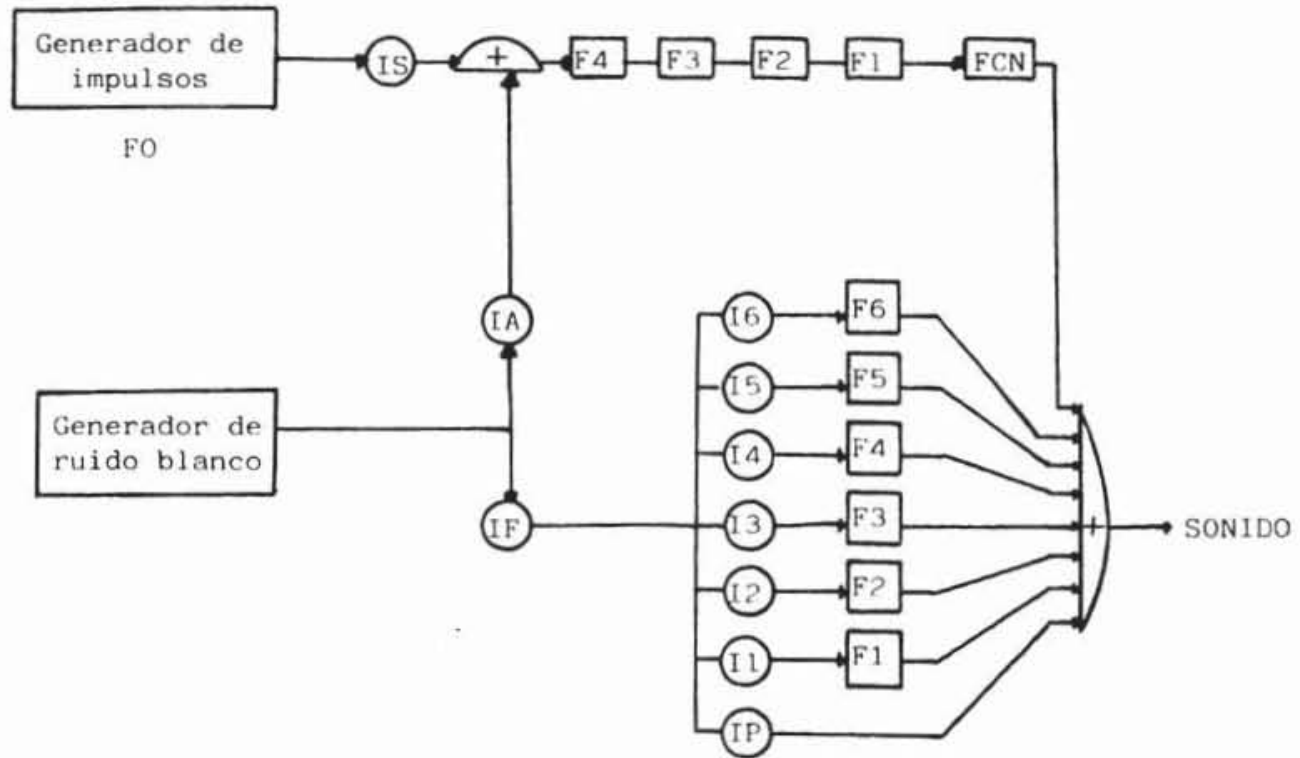

FO Frecuencia fundamental de sonoridad

IS Intensidad de sonoridad

IA Intensidad de aspiración

IF Intensidad de fricación

F1-F6 Frecuencia del primer formanteFrecuencia del sexto formante

FCN Frecuencia del cero nasal

I1-I6 Intensidad del primer formanteIntensidad del sexto formante

IP Intensidad del paso subsidiario
(FO Fundamental frequency of voicing)

(AV Amplitude of voicing)

(AH Amplitude of aspiration)

(AF Amplitude of frication)

(F1-F6 First formant frequency-

Sixth formant frequency)

(FNZ Nasal zero frequency)

(A1-A6 First formant amplitude-

Sixth formant amplitude)

(AB Bypass path amplitude) ${ }^{9}$

Figura 3.-Sintetizador de Klatt ${ }^{10}$

8 También presenta una posibilidad alternativa de conexión solamente en paralelo (cfr. Klatt, art. cit., pág. 973, fig. 4).

- Sobre este último parámetro - del que no vamos a tratar por quedar fuera de nues. tro objeto- indica Klatt: «Also included in the parallel vocal tract model is a bypass path. The bypass path with amplitude control $\mathrm{AB}$ is present because the transfer functions for $[f, v, \diamond, \delta, p, b]$ containt no prominent resonant peaks, and the synthesizer should include a means of bypassing all of the resonators to produce a flat transfer functionw (art. cit., pág. 981). Por otra parte, el sintetizador permite también manipular los anchos de banda de los formantes, representados como $\mathrm{AB1}-\mathrm{AB} 4$ en las tablas 7,8 y 9 (págs. 50 51).

10 Cfr. Klatt, art. cit., pág. 975, figura 6. 
Además, la cavidad suplementaria que interviene en la articulación de las consonantes que nos ocupan, introduce antirresonancias en la función de transferencia. Por ello, para la sintesis de estos sonidos, se incluye también un antirresonador (representado como FCN en la figura 3).

Así, de todos los parámetros que permite variar el sintetizador, los que hay que tener en cuenta para las consonantes nasales son: con relación a la fuente, la intensidad de sonoridad, que depende de la frecuencia fundamental $^{11}$; en lo que se refiere al filtro, las frecuencias y anchos de banda de los formantes y la frecuencia del cero nasal.

El análisis y la síntesis nos han revelado los rasgos acústicos de las nasales: su duración y su intensidad de sonoridad, asi como las características que presentan sus formantes y las transiciones que las enlazan con la vocal siguiente.

\subsubsection{Duraciones}

En nuestros sonogramas hemos medido, en primer lugar, la porción de tiempo que dura la articulación de cada sonido. Apenas presenta diferencias si se compara el fonema bilabial con el alveolar, pero sí se aprecian entre estos dos y el palatal, siempre de duración más larga, puesto que posee unas transiciones muy acusadas hacia la vocal.

Por otra parte, $[\mathrm{m}]$ y $[\mathrm{n}]$ alcanzan a menudo una duración un poco mayor cuando van en posición explosiva, es decir, cuando constituyen la rama intensiva de una sílaba. Ello se explica por la tendencia del español a la sílaba libre ${ }^{12}$, en la que la fuerza articulatoria alcanza el máximo al comienzo y disminuye hacia el final.

En posición intervocálica no hemos observado diferencias constantes según la nasal forme sílaba con una vocal tónica o átona, como tampoco han aparecido en posición implosiva en función del carácter sordo o sonoro de la consonante siguiente. Éstos son los valores medios en nuestro análisis ${ }^{13}$ :

11 Las fuentes de fricación y de aspiración, naturalmente se encuentran cerradas.

12 Cfr. Malmberg, «La estructura silábica del español», en Estudios de Fonética hispánica, Madrid, CSIC, 1965, págs. 3-28.

18 Nuestros resultados corroboran en lo esencial las impresiones de Navarro Tomás: «En posición intervocálica, inmediatamente detrás de la vocal acentuada, paso, pala, las consonantes son más largas que en ninguna otra posición. Finales de sflaba interior, pasta, $a l b a$, son muy poco más cortas que intervocálicas. Separadas de la vocal acentuada, posición, olivar, son asimismo un poco más cortas que en contacto con dicha vocal. En posición inicial o final absoluta, sabio, jamás, etc., su articulación suele ser relativamente larga; pero la parte de su sonido propiamente perceptible es siempre breve» (Manual de pronunciación española [23:" ed.], Madrid, CSIC, 1989, pág. 204). 


\section{TABLA 1}

\section{Duración de las consonantes nasales}

\begin{tabular}{|c|c|c|c|}
\hline & [m] & [n] & [n] \\
\hline $\begin{array}{llllll}\text { Inicial } & \ldots & \ldots & \ldots & \ldots & \ldots\end{array}$ & 10 & 9,5 & 13,5 \\
\hline $\begin{array}{llllll}\text { Intervocálica } & \ldots & \ldots & \ldots\end{array}$ & 9,5 & 8,7 & 16,2 \\
\hline Implosiva $\ldots \ldots \ldots c c c c$ & 10,2 & 9,1 & \\
\hline Duración media $\ldots \ldots$ & 9,9 & 9,1 & 14,8 \\
\hline
\end{tabular}

En posición implosiva, si la nasal va seguida de una oclusiva oral, al comparar la duración de ambas es fácil entrever una clara tendencia : en el caso de las sonoras, la nasal es siempre más larga que la oral (aproximadamente el doble), mientras que con las sordas sucede lo contrario: la duración de la oral rebasa en unas 2 c. s. como media a la de la nasal. Las duraciones de la oclusiva en las combinaciones que se transcriben son las siguientes:

\section{TABLA 2}

Duración de las oclusivas precedidas de nasal

Ocl. bilabial

$\overline{\frac{[-\mathrm{mb}-]}{5,6}} \frac{[-\mathrm{mp}-]}{11,4} \frac{[-\mathrm{nd}-]}{5,5} \frac{[-\mathrm{nt}-]}{11,9}$

Ocl. velar

$\frac{[-n g-]}{5,3} \frac{[-n k-]}{12,7}$

c. $\mathbf{s}$.

Ya Navarro Tomás ${ }^{14}-\mathrm{y}$ aún antes Menéndez Pidal ${ }^{15}$ - señalaron este hecho en lo que se refiere a las oclusivas sonoras: en los grupos $-m b-$, $-n d-$ y - $n g$ - "las consonantes $b, d, g$ van cediendo el campo a la nasal anterior" y se produce "un marcado alargamiento de la articulación nasal a costa de la consonante siguiente" ${ }^{16}$. Malmberg, por su parte, explica este fenómeno como

14 «La metafonía vocálica y otras teorías del señor Colton», Revista de Filología española, X, 1923, págs. 26-56.

15 Manual de Gramática bistórica española (14." ed.), Madrid, Espasa Calpe, 1973.

16 Sus datos son muy próximos a los nuestros: «En una misma inscripción quimográfica -indica Navarro- la duración de la $n$ de venta, por ejemplo, ha sido 7 centésimas de segundo y la de la $t, 10$, mientras que en la palabra venda la $n$ ha durado 10 y la $d 5$. En campo, la $m$ ha durado 7 y la $p$ 11; en ambos, la $m 9$ y la $b$ 5,5. En tenca, la $n$ ha durado 7,5 y la $k 10,5$; en tenga, la $n 9$ y la 8 6m (art. cit., pág. 43). 
una consecuencia de la tendencia del español a la sílaba abierta - a la cual venimos aludiendo-, que conduce a una "alteración del lugar del corte silábico" ${ }^{17}$ en estos casos en que la consonante traba la sílaba, lo cual justifica el resultado $m b>m$ (palumba $>$ paloma, también $>$ tamién), así como las etapas intermedias que es posible encontrar en la lengua popular ${ }^{18}$.

\subsubsection{Intensidad de sonoridad. Frecuencia fundamental}

La frecuencia fundamental de una onda sonora origina un tren de impulsos que determinan la sonoridad. La intensidad de cada impulso viene dada por el parámetro que llamamos intensidad de sonoridad. Los valores que puede alcanzar están comprendidos en una escala que va de $0 \mathrm{db}$. cuando la fuente de sonoridad se encuentra cerrada a unos $80 \mathrm{db}$. en los sonidos más sonoros.

En nuestra sintesis, las nasales presentan un fundamental de $122 \mathrm{~Hz}$. (FO en las tablas 7,8 y 9) y una intensidad de sonoridad (IS en las tablas) de 60 db. ${ }^{19}$.

\subsubsection{Los formantes de la tensión nasal}

El sintetizador presenta en su rama conectada en serie varios resonadores -que se comportan como otros tantos filtros- capaces de simular los sonidos periódicos, por medio de los cuales es posible manipular sus formantes.

17 Op. cit., pág. 23.

18 Los ejemplos de la lengua popular que indica Malmberg son [ámos] por ambos y [béna] por venda. La alteración del lugar del corte silábico se realizó pasando por una etapa intermedia: [ $\mathrm{a}^{\mathrm{m}} \mid \mathrm{m}^{\mathrm{b}} \mathrm{os}$ ], [bé ${ }^{\mathrm{n}} \mid \mathrm{n}^{\mathrm{a}} \mathrm{a}$ ]. Estos cambios, como se sabe, fueron explicados por Menéndez Pidal en Orígenes del español. Estudio lingüístico de la Peninsula Ibérica hasta el siglo XI (7:" ed.), Madrid, Espasa Calpe, 1972, pág. 309, y por García de Diego en Manual de Dialectología española (3." ed.), Madrid, Centro iberoamericano de cooperación, 1978, pág. 14, como efectos de la influencia de un sustrato osco-umbro. Malmberg, sin embargo, propone esta explicación basada en la tendencia del español a la sílaba libre: «Un hecho de fonética plenamente viviente todavía hoy en la mayor parte de las hablas populares de España y de América, ¿debe verdaderamente explicarse por la influencia de un antiguo dialecto itálico mal conocido? A mí me parece evidente que la antigua alteración lumbu $>$ lomo y el moderno cambio también $>$ tamién son reflejos de la misma tendencia fónica, que actúa a través de la historia de los dialectos españoles desde los orígenes hasta nuestros días" (op. cit., págs. 23-24).

19 Téngase en cuenta que entre las consonantes, las nasales ocupan el segundo lugar en la escala de sonoridad después de las líquidas, que presentan las características acústicas más próximas a las de las vocales. 


\subsubsection{Intensidad}

La configuración en serie no permite el filtrado de la intensidad de cada formante ${ }^{20}$. Según Klatt, "The amplitudes of the formant peaks generated by the parallel vocal tract model have been constrained such that, if A1 to A5 are all set to $60 \mathrm{db}$, the transfer function will approximate that found in the cascade model" ${ }^{21}$. Sin embargo, es posible variar la intensidad de los formantes alterando sus anchos de banda (AB1, AB2 y AB3 en las tablas 7, 8 y 9), puesto que existe una relación inversamente proporcional entre ambos parámetros: "If a formant bandwidth is doubled, that formant peak is reduced in amplitude by $6 \mathrm{db}$. If the bandwidth is halved, the peak is increased by $6 \mathrm{db}$ " ${ }^{22}$. Esto explica la escasa anchura de banda del primer formante nasal $(40 \mathrm{~Hz}$.), de intensidad considerablemente más alta que los formantes superiores :

TABLA 3

Anchos de banda de los formantes

\begin{tabular}{|c|c|c|c|}
\hline & {$[\mathrm{m}]$} & [n] & [n] \\
\hline $\mathrm{AB} 1 \ldots \ldots \ldots$ & 40 & 40 & 40 \\
\hline $\mathrm{AB} 2 \ldots \ldots \ldots$ & 200 & 300 & 300 \\
\hline $\mathrm{AB} 3 \ldots \ldots \ldots \ldots$ & 200 & 300 & 300 \\
\hline
\end{tabular}

\subsubsection{Frecuencia}

En cuanto a la frecuencia (cfr. tablas 7,8 y 9), la del primer formante se sitúa en nuestro análisis en unos $480 \mathrm{~Hz}$.; la del segundo en torno a los 1.000 ; la del tercero algo por encima de los 2.000 , y la del cuarto sobre los $3.300 \mathrm{~Hz}{ }^{23}$ :

20 La síntesis en paralelo, sin embargo, sí lo permite, tal como se ve en la figura 3.

21 Art. cit., pág. 982.

22 Ibid.

$23 \mathrm{La}$ frecuencia de los tres primeros formantes, como es conocido, varía notablemente de unos sonidos a otros, en tanto que la de los formantes superiores presenta una estabilidad considerable: «The frequencies of the lowest three formants vary substantialy with changes to articulation (e. g., the observed range of $F 1$ is from about 180 to $750 \mathrm{~Hz}$, of F2 is 600 to $2.300 \mathrm{~Hz}$, and of F3 is 1.300 to $3.100 \mathrm{~Hz}$ for a typical male talker). The frequencies and bandwidths of the 4th and 5th formant resonators do not vary as much and could be held constant with little decrement in output sound quality» (Klatt, art. cit., pág. 980). Así, en el caso de las consonantes nasales, las frecuencias que varían de unas a otras son las del segundo y tercer formantes. 


\section{TABLA 4}

Frecuencias de los formantes

\begin{tabular}{|c|c|c|c|}
\hline & {$[\mathrm{m}]$} & [n] & [n] \\
\hline $\begin{array}{llllll} & F 1 & \ldots & \ldots & \ldots & \ldots\end{array}$ & 480 & 480 & 480 \\
\hline 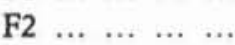 & 1.052 & 1.052 & 1.004 \\
\hline $\begin{array}{llllll}\text { F3 } & \ldots & \ldots & \ldots & \ldots\end{array}$ & 2.320 & 2.240 & 2.400 \\
\hline $\mathrm{F} 4 \quad \ldots \ldots \ldots \ldots$ & 3.296 & 3.296 & 3.296 \\
\hline
\end{tabular}

Con relación al primero, según Delattre ${ }^{24}$, la aparición en el sonograma de un formante bajo de la frecuencia aproximada de este primer formante nasal y la presencia de una mancha vertical inarmónica (como la turbulencia característica de las fricativas o como la barra de explosión de las oclusivas orales) son mutuamente excluyentes, razón por la cual no aparece tal mancha en las nasales (como tampoco aparece un formante bajo en las oclusivas y fricativas). Esta mancha inarmónica refleja una salida violenta del aire hacia el exterior por medio de una explosión (en las oclusivas) o de una fricción (en las fricativas). El formante de frecuencia baja, sin embargo, se relaciona con un flujo de la corriente de aire a través de una constricción suficientemente amplia como para permitir su salida suave hacia el exterior.

\subsubsection{Frecuencia del cero nasal}

En cualquier onda sonora es posible distinguir unos puntos en los que la frecuencia alcanza los valores más altos (que se llaman picos o polos) y otros en los que presenta los valores mínimos (que se denominan valles o ceros). En la articulación de los sonidos nasales, la intervención de un segundo resonador introduce polos y ceros adicionales. Por ello, como hemos visto, para simular la resonancia nasal es necesario un resonador más que filtra la frecuencia del cero nasal (FCN en las tablas 7, 8 y 9). Ésta es de unos $250 \mathrm{~Hz}$. en los sonidos no nasalizados y se eleva a unos $450 \mathrm{~Hz}$. cuando aparece la nasalidad.

24 «From Acoustic Cues to Distinctive Features», Phonetica, 18, 1968, págs, 208-209. 


\subsubsection{Las transiciones}

En nuestros sonogramas hemos estudiado también las transiciones que enlazan -en el interior de cada sílaba - la consonante con la vocal precedente o siguiente.

\subsubsection{Duración}

Cuando la nasal se encuentra en posición explosiva -inicial o intervocálica- presentan una duración considerablemente mayor las transiciones del fonema linguopalatal, en tanto que apenas hay diferencias entre las del bilabial y las del alveolar ${ }^{25}$.

En posición implosiva, la duración de la transición es algo mayor y ello encuentra de nuevo su explicación - a pesar de lo exiguo de la diferenciaen la tendencia del español a la sílaba libre: las consonantes que traban sílaba, tienden a acortar su duración en la misma medida en que la transición adquiere relieve y se prolonga algo temporalmente ${ }^{26}$. Éstos son los valores en nuestro análisis:

\section{TABLA 5}

Duración de las transiciones

\begin{tabular}{|c|c|c|c|}
\hline & [m] & [n] & [n] \\
\hline Inicial $\ldots \ldots \ldots \ldots$ & 0,85 & 0,80 & 3,65 \\
\hline $\begin{array}{lllll}\text { Intervocálica } & \ldots & \ldots & \ldots\end{array}$ & 0,85 & 0,75 & 3,65 \\
\hline Implosiva $\quad \ldots \ldots \ldots c c c c$ & 1 & 1,2 & \\
\hline Duración media $\ldots \ldots \ldots$ & 0,9 & 0,9 & 3,65 \\
\hline
\end{tabular}

\subsubsection{Velocidad}

En cuanto a la velocidad, las transiciones de las nasales - como las de las oclusivas- son rápidas, rapidez que es inherente a un momento de cierre de los órganos articulatorios durante la tensión ${ }^{27}$.

${ }^{25}$ Medimos siempre -como es habitual- la duración de la transición hacia la vocal con la que la consonante forma silaba.

${ }^{26}$ Nos referimos sólo, naturalmente, a las transiciones de [m] y de [n], puesto que la nasal linguopalatal no puede aparecer en esta posición silábica.

${ }_{27}$ Con relación al tempo, como es conocido, las transiciones pueden ser lentas (sua. 


\subsubsection{Discontinuidad}

Por otra parte, según Delattre ${ }^{28}$, en las consonantes nasales, la unión de sus formantes con las transiciones de la vocal siguiente puede ser discontinua : así como en una consonante líquida, por ejemplo, los formantes se prolongan sin solución de continuidad hasta alcanzar la frecuencia de la vocal siguiente, en una nasal, sin embargo, puede apreciarse en el sonograma una interrupción entre el final del formante de la consonante y el comienzo de la transición hacia el formante de la vocal. Esta interrupción se corresponde en lo articulatorio con el paso de la corriente de aire desde la vía nasal (en el instante en que la boca se encuentra cerrada) hacia la vía bucal (cuando la boca momentáneamente se abre).

\subsubsection{Dirección}

La dirección de las transiciones de los tres primeros formantes hacia su locus es, como se sabe, un índice tanto del modo como del lugar de articulación de la consonante.

La transición del primer formante es fundamentalmente índice del modo de articulación, puesto que el incremento de su frecuencia está en relación directa con el carácter vocalizado de la consonante ${ }^{20}$. Así, en las nasales presenta un comienzo bajo - pero algo más elevado que en las oclusivas orales-, y una intensidad considerable, que corresponde a su carácter sonoro, puesto que según Delattre, "plus la transition du premier formant est faible, plus la consonne est sourde" ${ }^{30}$. Su dirección es recta en todos los casos.

ves) o rápidas (abruptas), y es posible establecer una jerarquía de menor a mayor rapidez en función del carácter más o menos vocalizado de la consonante: conforme se avanza hacia la abertura, más lentas se van haciendo las transiciones. Como indica Pierre Delattre, «plus les transitions sont lentes, plus la consonne est vocalisée» («Le jeu des transitions de formants et la perception des consonnesn, Proceedings of 4th International Congress of Phonetic Sciences, The Hague, Mouton, 1962, pág. 416).

${ }_{28}$ «From Acoustic Cues to Distinctive Features», pág. 211.

29 Sin embargo, indica Delattre que también permite distinguir -en las lenguas en que hay fonemas de uno y otro tipo- entre dos zonas de articulación, la bucal y la faríngea: «Lorsque la constriction consonantique est dans la moitié antérieure du pavillon (dès lèvres au palais mou) la transition du premier formant est négative par rapport à la voyelle neutre (autrement dit elle commence inicielment plus bas que 500 cycles environ), et lorsque la constriction consonantique est dans la moitié postérieure du pavillon (dans le pharynx) la transition du premier formant est positive par rapport à la voyelle neutre (autrement dit elle commerce plus haut que 500 cycles environ)» ( Le jeu des transitions de formants et la perception des consonnes", pág. 415).

so Ibid., pág. 417. 
Las transiciones del segundo y tercer formantes, por su parte, son índices del lugar articulatorio. En la nasal bilabial convergen virtualmente hacia una frecuencia baja y son negativas. Con las vocales posteriores generalmente no hay transición del tercer formante. En la alveolar, tienden hacia una frecuencia intermedia, con ausencia de transición con la vocal central, con dirección negativa con las vocales anteriores y positiva con las posteriores. En la palatal, tienden hacia una frecuencia alta, con dirección positiva, excepto la del tercer formante con vocales posteriores, que es negativa :

TABLA 6

Dirección de las transiciones

\begin{tabular}{|c|c|c|c|c|c|c|c|c|c|c|}
\hline & & & & & & & & & & \\
\hline & $\mathrm{T} 2$ & T3 & $\mathrm{T} 2$ & $\mathrm{~T} 3$ & T2 & T3 & T2 & T3 & T2 & T3 \\
\hline $\mathrm{m} \ldots \ldots \ldots$ & - & 一 & - & - & - & - & - & $=$ & - & $=$ \\
\hline $\mathrm{n} \ldots \ldots \ldots$ & - & - & - & - & $=$ & $=$ & + & - & + & - \\
\hline $\mathrm{n} \ldots \ldots \ldots$ & + & + & + & + & + & + & + & - & + & - \\
\hline
\end{tabular}

Con respecto a la correspondencia fisiológica de estos hechos acústicos, indica Delattre ${ }^{31}$ que si consideramos el tracto vocal como un tubo único desde la glotis hasta los labios, el locus de las transiciones del segundo y tercer formantes se relaciona con una constricción de los órganos articulatorios en un punto determinado de esa trayectoria: a una transición con un locus bajo le corresponde un contacto en la zona más anterior, mientras que una oclusión en la región más posterior le es inherente a un locus alto ${ }^{32}$. Así, en las realizaciones en posición implosiva que hemos analizado, la bilabial repite el esquema de [m]; la velar y la palatalizada presentan las caracteristicas de [n] y los alófonos dentales muestran transiciones de dirección positiva.

31 «From Acoustic Cues to Distinctive Features», pág. 207.

32 Ello permite establecer características acústicas de un mismo lugar articulatorio en varios modos de articulación: según Liberman, Delattre, Cooper y Gertsman, «a single second-formant transition can serve for $/ \mathrm{p} /, / \mathrm{b} /$ and $/ \mathrm{m} /$, another for $/ \mathrm{t} /, / \mathrm{d} /$ and $/ \mathrm{n} /$ and a third for $/ \mathrm{k} /, / \mathrm{g} /$ and $/ \mathrm{g} /$. Thus, three transitions are sufficient to classify nine speech sounds into three categories of three sounds each, and to enable a listener, with some degree of reability, to distinguish among the categories. This arrangement of the sounds corresponds to a commonly accepted linguistic classification according to the place of articulation, in terms of which /p-b-m/ are bilabial, /t-d-n/ are linguo-alveolar and / $\mathrm{k}-\mathrm{g}-\mathrm{n} / \mathrm{are}$ linguo-velar» ( $*$ The Role of Consonant-vowel Transitions in the Perception of the Stop and Nasal Consonants», Psychological Monographe, 379, pág. 329). 


\subsubsection{Recapitulación}

Si recapitulamos todos los datos, tenemos ya los parámetros de las consonantes nasales españolas (véase figura 3 ):

\section{TABLA 7}

Parámetros de $[m]$ en la silaba [ma]

\begin{tabular}{|c|c|c|c|c|c|c|c|c|c|c|c|c|c|c|c|c|c|}
\hline IS & IF & IA & I1 & I2 & I3 & I4 & I5 & IP & F1 & F2 & F3 & F4 & $\mathrm{AB} 1$ & AB2 & AB3 & $\mathrm{FCN}$ & FO \\
\hline IS & IF & IA & I1 & I2 & I3 & I4 & Is & IP & F1 & F2 & F3 & F4 & $\mathrm{AB} 1$ & AB2 & $\mathrm{AB3}$ & $\mathrm{FCN}$ & FO \\
\hline \multicolumn{18}{|c|}{$[\mathrm{m}]$} \\
\hline 60 & 0 & 0 & 60 & 60 & 60 & 60 & 60 & 0 & 480 & 1.052 & 2.320 & 3.296 & 40 & 200 & 200 & 448 & 122 \\
\hline 60 & 0 & 0 & 60 & 60 & 60 & 60 & 60 & 0 & 480 & 1.052 & 2.320 & 3.296 & 40 & 200 & 200 & 448 & 22 \\
\hline 60 & 0 & 0 & 60 & 60 & 60 & 60 & 60 & 0 & 480 & 1.052 & 2.320 & 3.296 & 40 & 200 & 200 & 448 & 122 \\
\hline 60 & 0 & 0 & 60 & 60 & 60 & 60 & 60 & 0 & 480 & 1.052 & 2.320 & 3.296 & 40 & 200 & 200 & 448 & 122 \\
\hline 60 & 0 & 0 & 60 & 60 & 60 & 60 & 60 & 0 & 480 & 1.052 & 2.320 & 3.296 & 40 & 200 & 200 & 448 & 122 \\
\hline 60 & 0 & 0 & 60 & 60 & 60 & 60 & 60 & 0 & 480 & 1.052 & 2.320 & 3.296 & 40 & 200 & 200 & 448 & 122 \\
\hline 60 & 0 & 0 & 60 & 60 & 60 & 60 & 60 & 0 & 480 & 1.052 & 2.320 & 3.296 & 40 & 200 & 200 & 448 & 122 \\
\hline 60 & 0 & 0 & 60 & 60 & 60 & 60 & 60 & 0 & 480 & 1.052 & 2.320 & 3.296 & 40 & 200 & 200 & 448 & 122 \\
\hline 60 & 0 & 0 & 60 & 60 & 60 & 60 & 60 & 0 & 480 & 1.052 & 2.320 & 3.296 & 40 & 200 & 200 & 448 & 122 \\
\hline \multicolumn{18}{|c|}{ [a] } \\
\hline 60 & 0 & 0 & 60 & 60 & 60 & 60 & 60 & 0 & 472 & 980 & 2.240 & 3.296 & 106 & 120 & 146 & 388 & 120 \\
\hline 59 & 0 & 0 & 60 & 60 & 60 & 60 & 60 & 0 & 572 & 1.116 & 2.256 & 3.296 & 88 & 120 & 148 & 324 & 118 \\
\hline 59 & 0 & 0 & 60 & 60 & 60 & 60 & 60 & 0 & 676 & 1.220 & 2.272 & 3.296 & 82 & 120 & 150 & 280 & 116 \\
\hline 59 & 0 & 0 & 60 & 60 & 60 & 60 & 60 & 0 & 700 & 1.284 & 2.288 & 3.296 & 86 & 120 & 150 & 256 & 114 \\
\hline 58 & 0 & 0 & 60 & 60 & 60 & 60 & 60 & 0 & 696 & 1.300 & 2.288 & 3.296 & 104 & 120 & 150 & 248 & 113 \\
\hline 53 & 0 & 36 & 60 & 60 & 60 & 60 & 60 & 0 & 684 & 1.300 & 2.288 & 3.296 & 128 & 120 & 148 & 248 & 112 \\
\hline 0 & 0 & 44 & 60 & 60 & 60 & 60 & 60 & 0 & 664 & 1.308 & 2.288 & 3.296 & 66 & 118 & 146 & 248 & 111 \\
\hline
\end{tabular}

TABLA 8

Parámetros de [n] en la silaba [na]

$\begin{array}{cccccccccccccccccc}\text { IS } & \text { IF } & \text { IA } & \text { I1 } & \text { I2 } & \text { I3 } & \text { I4 } & \text { I5 } & \text { IP } & \text { F1 } & \text { F2 } & \text { F3 } & \text { F4 } & \text { AB1 } & \text { AB2 } & \text { AB3 } & \text { FCN } & \text { FO } \\ \text { [n] } & & & & & & & & & & & & & & & & & \\ 60 & 0 & 0 & 60 & 60 & 60 & 60 & 60 & 0 & 480 & 1.052 & 2.240 & 3.296 & 40 & 300 & 300 & 448 & 122 \\ 60 & 0 & 0 & 60 & 60 & 60 & 60 & 60 & 0 & 480 & 1.052 & 2.240 & 3.296 & 40 & 300 & 300 & 448 & 122 \\ 60 & 0 & 0 & 60 & 60 & 60 & 60 & 60 & 0 & 480 & 1.052 & 2.240 & 3.296 & 40 & 300 & 300 & 448 & 122 \\ 60 & 0 & 0 & 60 & 60 & 60 & 60 & 60 & 0 & 480 & 1.052 & 2.240 & 3.296 & 40 & 300 & 300 & 448 & 122 \\ 60 & 0 & 0 & 60 & 60 & 60 & 60 & 60 & 0 & 480 & 1.052 & 2.240 & 3.296 & 40 & 300 & 300 & 448 & 122 \\ 60 & 0 & 0 & 60 & 60 & 60 & 60 & 60 & 0 & 480 & 1.052 & 2.240 & 3.296 & 40 & 300 & 300 & 448 & 122 \\ 60 & 0 & 0 & 60 & 60 & 60 & 60 & 60 & 0 & 480 & 1.052 & 2.240 & 3.296 & 40 & 300 & 300 & 448 & 122 \\ & & & & & & & & & & & & & & & & & \\ \text { [a] } & & & & & & & & & & & & & & & & & \\ 60 & 0 & 0 & 60 & 60 & 60 & 60 & 60 & 0 & 400 & 1.388 & 2.592 & 3.296 & 70 & 214 & 170 & 432 & 120 \\ 59 & 0 & 0 & 60 & 60 & 60 & 60 & 60 & 0 & 532 & 1.348 & 2.464 & 3.296 & 68 & 174 & 162 & 348 & 118 \\ 59 & 0 & 0 & 60 & 60 & 60 & 60 & 60 & 0 & 668 & 1.324 & 2.368 & 3.296 & \mathbf{7 2} & 144 & 154 & 288 & 116 \\ 59 & 0 & 0 & 60 & 60 & 60 & 60 & 60 & 0 & 700 & 1.300 & 2.304 & 3.296 & 84 & 126 & 150 & 260 & 114 \\ 58 & 0 & 8 & 60 & 60 & 60 & 60 & 60 & 0 & 696 & 1.300 & 2.288 & 3.296 & 104 & 120 & 150 & 248 & 113 \\ 53 & 0 & 36 & 60 & 60 & 60 & 60 & 60 & 0 & 684 & 1.300 & 2.288 & 3.296 & 128 & 120 & 148 & 248 & 112 \\ 0 & 0 & 44 & 60 & 60 & 60 & 60 & 60 & 0 & 664 & 1.308 & 2.288 & 3.296 & 66 & 118 & 146 & 248 & 111\end{array}$


TABLA 9

Parámetros de $[v]$ en la silaba $[v a]$

$\begin{array}{cccccccccccccccccc}\text { IS } & \text { IF } & \text { IA } & \text { I1 } & \text { I2 } & \text { I3 } & \text { I4 } & \text { I5 } & \text { IP } & \text { F1 } & \text { F2 } & \text { F3 } & \text { F4 } & \text { AB1 } & \text { AB2 } & \text { AB3 } & \text { FCN } & \text { FO } \\ \text { Ib] } & & & & & & & & & & & & & & & & & \\ 60 & 0 & 0 & 60 & 60 & 60 & 60 & 60 & 0 & 480 & 1.004 & 2.400 & 3.296 & 40 & 300 & 300 & 448 & 122 \\ 60 & 0 & 0 & 60 & 60 & 60 & 60 & 60 & 0 & 480 & 1.004 & 2.400 & 3.296 & 40 & 300 & 300 & 448 & 122 \\ 60 & 0 & 0 & 60 & 60 & 60 & 60 & 60 & 0 & 480 & 1.004 & 2.400 & 3.296 & 40 & 300 & 300 & 448 & 122 \\ 60 & 0 & 0 & 60 & 60 & 60 & 60 & 60 & 0 & 480 & 1.004 & 2.400 & 3.296 & 40 & 300 & 300 & 448 & 122 \\ 60 & 0 & 0 & 60 & 60 & 60 & 60 & 60 & 0 & 480 & 1.004 & 2.400 & 3.296 & 40 & 300 & 300 & 448 & 122 \\ 60 & 0 & 0 & 60 & 60 & 60 & 60 & 60 & 0 & 480 & 1.004 & 2.400 & 3.296 & 40 & 300 & 300 & 448 & 122 \\ 60 & 0 & 0 & 60 & 60 & 60 & 60 & 60 & 0 & 480 & 1.004 & 2.400 & 3.296 & 40 & 300 & 300 & 448 & 122 \\ 60 & 0 & 0 & 60 & 60 & 60 & 60 & 60 & 0 & 480 & 1.004 & 2.400 & 3.296 & 40 & 300 & 300 & 448 & 122 \\ 60 & 0 & 0 & 60 & 60 & 60 & 60 & 60 & 0 & 480 & 1.004 & 2.400 & 3.296 & 40 & 300 & 300 & 448 & 122 \\ 60 & 0 & 0 & 60 & 60 & 60 & 60 & 60 & 0 & 480 & 1.004 & 2.400 & 3.296 & 40 & 300 & 300 & 448 & 122 \\ 60 & 0 & 0 & 60 & 60 & 60 & 60 & 60 & 0 & 480 & 1.004 & 2.400 & 3.296 & 40 & 300 & 300 & 448 & 122 \\ 60 & 0 & 0 & 60 & 60 & 60 & 60 & 60 & 0 & 480 & 1.004 & 2.400 & 3.296 & 40 & 300 & 300 & 448 & 122 \\ 60 & 0 & 0 & 60 & 60 & 60 & 60 & 60 & 0 & 480 & 1.004 & 2.400 & 3.296 & 40 & 300 & 300 & 448 & 122 \\ & & & & & & & & & & & & & & & & & \\ \text { [a] } & & & & & & & & & & & & & & & & \\ 60 & 0 & 0 & 60 & 60 & 60 & 60 & 60 & 0 & 400 & 2.052 & 2.576 & 3.296 & 70 & 214 & 150 & 388 & 120 \\ 59 & 0 & 0 & 60 & 60 & 60 & 60 & 60 & 0 & 532 & 1.900 & 2.400 & 3.296 & 68 & 174 & 150 & 324 & 118 \\ 59 & 0 & 0 & 60 & 60 & 60 & 60 & 60 & 0 & 668 & 1.772 & 2.256 & 3.296 & 72 & 144 & 150 & 280 & 116 \\ 59 & 0 & 0 & 60 & 60 & 60 & 60 & 60 & 0 & 700 & 1.652 & 2.176 & 3.296 & 84 & 126 & 150 & 256 & 114 \\ 58 & 0 & 8 & 60 & 60 & 60 & 60 & 60 & 0 & 696 & 1.556 & 2.144 & 3.296 & 104 & 120 & 150 & 248 & 113 \\ 53 & 0 & 36 & 60 & 60 & 60 & 60 & 60 & 0 & 684 & 1.460 & 2.192 & 3.296 & 128 & 120 & 148 & 248 & 112 \\ 0 & 0 & 44 & 60 & 60 & 60 & 60 & 60 & 0 & 664 & 1.372 & 2.240 & 3.296 & 66 & 118 & 146 & 248 & 111\end{array}$

\subsection{INDICES DEL MODO Y DEL LUGAR DE ARTICULACIÓN}

En cuanto al peso de cada uno de estos parámetros como índices del modo y del lugar articulatorio, hay acuerdo general en que la aparición de la resonancia es índice del modo de articulación nasal, junto con una intensidad global débil (excepto en el caso del primer formante), hasta el punto de que en un sonograma ennegrecido en grado normal apenas se destacan las regiones donde hay concentración de energía: es necesario que las vocales más intensas de la secuencia queden distorsionadas para que aparezcan visibles en grado suficiente los formantes de la tensión nasal.

Respecto a los indices del lugar articulatorio, Liberman, Delattre, Cooper y Gerstman ${ }^{33}$ realizaron pruebas de reconocimiento de espectrogramas artificiales reconvertidos en sonido, en los que se presentaban los mismos formantes de tensión nasal en las tres consonantes inglesas $/ \mathrm{m}, \mathrm{n}, \mathrm{n} /{ }^{34}$. Como los oyentes eran capaces de distinguir unas de otras, concluyeron que el ín-

$3 s$ Art. cit.

34 Las frecuencias de estos formantes eran de $240 \mathrm{~Hz}$., $1.020 \mathrm{~Hz}$. y $2.460 \mathrm{~Hz}$. 
dice del lugar articulatorio se encuentra en las transiciones, en tanto que la resonancia es índice del modo de articulación: "It is possible that the distinctions among these three classes $[/ \mathrm{p}-\mathrm{t}-\mathrm{k} /, / \mathrm{b}-\mathrm{d}-\mathrm{g} / \mathrm{y} / \mathrm{m}-\mathrm{n}-\mathrm{n} /]$ are effectively cued by a limited number of acoustic markers, such as voice bar and nasal resonance, each of wich is constant within its class and characteristic of a manner, rather than a place of articulation" ${ }^{35}$.

Sin embargo, estos autores admiten la posibilidad de que la frecuencia de los formantes contribuya en una pequeña medida al reconocimiento de los distintos lugares de articulación ${ }^{36}$. Sus ideas quedan corroboradas en varios trabajos posteriores ${ }^{37}$, y más adelante, el propio Delattre y Mermelstein ${ }^{38} \mathrm{com}$ prueban de nuevo - tanto en fragmentos de habla sintética como de habla natural- la exigua importancia de los formantes de tensión nasal para distinguir los diferentes fonemas nasales ${ }^{39}$.

Algunos admiten, no obstante, la posibilidad de que exista en la distribución de los formantes a lo largo de la columna del espectro algún rasgo que se repita de modo sistemático - con independencia de los hablantes y de los contornos vocálicos - en todas las realizaciones de un sonido, y permita así diferenciar a unos de otros. Para Malécot, por ejemplo, la resonancia nasal es principalmente índice del modo de articulación, pero tiene también un cierto papel en la identificación de los lugares articulatorios. La mayor o menor relevancia de este papel —que, no obstante, para él corresponde primordialmente a las transiciones- depende de la posición que ocupe la consonante nasal en el interior de la silaba: "It is also clear that the nasal resonances serve primarily as class markers, differentiating nasals from plosives etc., but that they are not completely neutral with respect to place identification; they

\footnotetext{
3s Art. cit., pág. 330.

36 Asf lo indica Delattre cuando habla sobre las conclusiones que se alcanzaron en este trabajo: «La fréquence des formants de tenue nasale supérieurs à celui de $250 \mathrm{cps}$ joue un rôle dans la perception du lieu d'articulation - faible, mais certain» ( From Acoustic Cues to Distinctive Features», pág. 240).

37 K. Nakata, «ynthesis and Perception of Nasal Consonants», Journal of the Acous * tical Society of America, 31, 1959, págs. 661-666; M. Hecker, «Studies of Nasal Conso. nants with an Articulatory Speech Synthesizer», Journal of the Acoustical Society of America, 34, 1962, págs. 179-187, y J. M. Pickett, «Some Acoustic Cues for Synthesis of the /n-d/ Distinction», Journal of the Acoustical Society of America, 38, 1965, págs. 474-477.

38 Delattre, «From Acoustic Cues to Distinctive Features», y Mermelstein, «On Detecting Nasals in Continouos Speech», Journal of the Acoustical Society of America, 61, 1977, págs. 581-587.

39 Según Delattre, contribuyen a la percepción de la nasalidad más por ser discontinuos que por las frecuencias a las que se encuentran: «the low murmur is the only im. portant one; the two higher ones are not distinctive by their frequency but only by their discontinuity $[\ldots]$ the role of nasal murmurs as place cues is negligible. In synthesis, they contribute to the perception of nasality by being discontinouos, regardless of their frequency» («From Acoustic Cues to Distinctive Features», pág. 211).
} 
contain a small amount of place information, almost negligible in initial position but somewhat more important in terminal position" 40 .

House $^{41}$, por su parte, indica - a partir de la síntesis de los sonidos nasales / $\mathrm{m}, \mathrm{n}, \mathrm{n} /$ por medio del análogo eléctrico de la cavidad bucal- la importancia del segundo formante para distinguir la bilabial frente a la alveolar y a la velar, que a su vez pueden diferenciarse entre sí por un formante superior a los $3.000 \mathrm{cps}$.

También Fant ${ }^{42}$ reconoce una cierta importancia a la resonancia como índice del lugar de articulación, aunque en su opinión la función más importante a este respecto corresponde a las transiciones.

Fujimura admite junto a éstas un rasgo más -el antiformante- que permite diferenciar los distintos sonidos nasales: "Although it is possible within the class of nasals to separate $/ \mathrm{m} /, / \mathrm{n} /$ and $/ \mathrm{n} /$ on the basis of the location of the antiformant, there is no doubt that the formant transitions of the adjacent vowels often play a more important or even dominant role in the recognition of the individual nasals" ${ }^{43}$.

Blumstein y Stevens ${ }^{44}$, por su parte, reconocen la existencia de configuraciones espectrales características de cada lugar articulatorio, al margen del contorno fonético, de la posición en el interior de la sílaba o de los rasgos individuales del hablante ${ }^{45}$. Según ellos, el sistema auditivo está capacitado para asociar cada una de estas configuraciones a la percepción de un sonido lingüístico determinado, lo que ayuda al niño durante la etapa de adquisición del lenguaje a organizar estos sonidos del habla en sus clases naturales. En un trabajo posterior, Kurowski y el propio Blumstein ${ }^{46}$ precisan que la resonancia nasal y las transiciones se integran en un rasgo único para la percepción de cada lugar de articulación y vuelven sobre la misma idea algo más adelante: "the perceptual cues to place of articulation in nasal consonants reside in the relation between spectral properties of the murmur and trans-

40 A. Malécot, «Acoustic Cues for Nasal Consonants; an Experimental Study involving a Tape-splicing Thecnique», Language, 32, 1956, págs. 274-284.

4 «Analog Studies of Nasalization of Vowels», Journal of Speech and Hearing Disorders, 22, 1957, págs. 190-204.

2 Cfr. op. cit.

4s "Analysis of Nasal Consonants», Journal of the Acoustical Society of America, 34, 1962, págs. 1.865-1.875.

44 «Acoustic Invariance in Speech Production: Evidence from Measurements of the Spectral Characteristics of Stop Consonants», Journal of the Acoustical Society of America, 66, 1979, págs. 1.001-1.017.

45 La configuración espectral integra, naturalmente, todos los parámetros que aparecen en el espectro de un sonido. En el caso de las nasales, tanto la resonancia como las transiciones.

46 «Perceptual Integration of the Murmur and Formant Transitions for Place of Ar. ticulation in Nasal Consonants», Journal of the Acoustical Society of America, 76, 1984, págs. 383-390. 
itions immediately following the nasal release, rather than in either the murmur or the transitions alone" ${ }^{47}$. Estas propiedades integradas permiten distinguir la articulación labial, la alveolar o la velar tanto en las oclusivas orales como en las nasales y constituyen así una especie de invariante acústica que caracteriza un determinado lugar articulatorio a través de diferentes modos de articulación ${ }^{48}$.

Recasens, por otro lado, comprobó por medio de síntesis la contribución de ambos parámetros a la percepción del lugar articulatorio en las nasales del catalán. Su conclusión es que ambos rasgos acústicos funcionan como índices del lugar de articulación, aunque la mayor importancia de uno u otro varía según el sonido de que se trate: "While transitions are generally more powerfull cues than murmurs, their relative power differs across place categories. [...] Thus there appears to be a reciprocal relationship between the relative salience of the two sets of cues" 4 .

También Repp corrobora en lo esencial las ideas de Kurowski y Blumstein, sobre las que se basa, e indica el peso mayor de las transiciones con relación a este aspecto ${ }^{50}$.

Nuestros resultados, por último, muestran también que son las transiciones el índice más importante para diferenciar los distintos lugares articulatorios, si bien es cierto que la frecuencia de los formantes contribuye en una cierta medida a la distinción entre la nasal bilabial frente a la alveolar y frente a la palatal. Hemos llegado a esta conclusión tras aplicar una doble prueba de reconocimiento: nuestros informantes escucharon, en primer término, las secuencias [ama], [ana], [ana] —ordenadas al azar- sintetizadas con los valores paramétricos que indicamos en las tablas 7,8 y 9 , es decir, tomando en cuenta como posibles índices tanto las transiciones cuanto las frecuencias de los formantes; después oyeron de nuevo las mismas secuencias sintetizadas esta vez con valores de frecuencia comunes a los tres sonidos, o sea tomando sólo en cuenta como índices hipotéticos las transiciones ${ }^{51}$. La proporción de aciertos de la segunda prueba es del $95 \%$, lo que demuestra con claridad que las transiciones bastan a los oyentes para distinguir entre sí las tres consonantes nasales. Sin embargo, la primera prueba alcanza un tanto por ciento de aciertos aún algo más alto, que nos lleva a concluir que los distintos

\footnotetext{
47 «Acoustic Properties for Place of Articulation in Nasal Consonants», Journal of the Acoustical Society of America, 81, 1987, págs. 1.917-1.927.

48 Cfr. lo indicado más arriba, en la nota 32.

49 «Place Cues for Nasal Consonants with Special Reference to Catalan», Journal of the Acoustical Society of America, 73, 1983, págs. 1.351-1.352.

so Cfr. «Perception of the [m] - [n] Distinction in CV Syllables», Journal of the Acoustical Society of America, 79, 1986, págs. 1.996-1.997.

B1 Estos valores fueron de $480 \mathrm{~Hz}$., $1.036 \mathrm{~Hz}$. y $3.296 \mathrm{~Hz}$.
} 
lugares articulatorios se identifican mejor cuando se considera también la frecuencia de los formantes.

Los indices fisiológicos y físicos de las consonantes nasales que hemos analizado no son sino correlatos de los rasgos distintivos que estructuran sus oposiciones en la lengua. Su íntima interdependencia mutua refleja lo apretado del lazo que une lo acústico con lo articulatorio, y ambas dimensiones fonéticas, a su vez, con lo fonológico. 